\title{
Vertrauen ist menschlich
}

Ingolf $U$. Dalferth

Vertrauen ist menschlich. Das ist meine - nicht ganz - triviale These. Denn was meint «menschlich?? Das ist die Frage, die ich im Folgenden erörtern will. Ohne sie zu klären, bleibt dunkel, was die These besagt. Und solange das nicht klar ist, kann man weder für noch gegen sie sein.

\section{Vertrauen als Lebensphänomen}

1. Wer vertraut, vertraut konkret - in bestimmten Situationen, auf bestimmte Weise, in bestimmte Personen. Auch wer Vertrauen versteht, versteht es konkret - als etwas Bestimmtes, in bestimmtem Horizont, von bestimmtem Standpunkt aus. Ohne Horizont gibt es nichts, was sich verstehen ließe, und ohne Standpunkt gibt es keinen Horizont. Nur von einem Standpunkt aus und in dessen Horizont kann Vertrauen als etwas Bestimmtes verstanden werden. Standpunkte aber können wechseln, Horizonte variieren mit Standpunkten, und deshalb kann Vertrauen auf mehr als nur eine Weise als etwas Bestimmtes verstanden werden.

2. Der Horizont, in dem wir Vertrauen in der Regel zu verstehen suchen, ist das menschliche Leben. Wir fassen Vertrauen als ein Lebensphänomen in den Blick, ein Phänomen im Leben einzelner (individuelles Vertrauen) und im Leben von Gemeinschaften (soziales Vertrauen). Dass Vertrauen ein Phänomen des menschlichen Lebens ist, werden wenige bestreiten wollen, auch wenn nicht alle unter 〈vertrauen〉 das gleiche oder gar dasselbe verstehen. Um welches Phänomen es konkret geht, kann von Fall zu Fall mit guten Gründen strittig sein, und wie dieses Phänomen jeweils zu verstehen ist auch. Unstrittig aber ist meist, dass es um ein Lebensphänomen geht, und zwar um ein charakteristisches Phänomen des menschlichen Lebens.

3. Damit ist weder behauptet, dass es Vertrauen nicht auch in anderem, nichtmenschlichem Leben geben könne (etwa bei Tieren oder bei Gott), noch dass es menschliches Leben ohne Vertrauen nicht geben könnte (dass Vertrauen also ein notwendiger Zug menschlichen Lebens wäre). Menschliches Leben wird nicht immer und 
überall vertrauend gelebt, und es ist nicht ausgemacht, dass das, was mit ‘vertrauen` gemeint wird, auf menschliches Leben beschränkt ist.

\section{Menschliches Leben}

4. Nun ist die Wendung (menschliches Leben` allerdings mehrdeutig. Zum einen kann sie das Leben von Menschen meinen im Unterschied $\mathrm{zu}$ dem anderer Lebewesen (Leitdifferenz menschlich/nichtmenschlich), zum anderen ein menschliches Leben von Menschen im Unterschied zu einem unmenschlichen (Leitdifferenz menschlich/unmenschlich). Im ersten Fall geht es um die Menschheit bzw. das Menschsein des Menschen (das, was Menschen zu Menschen macht), im zweiten um das Menschlichsein bzw. die Menschlichkeit von Menschen (das, was Menschen menschlich macht). Beides kann unterschiedlich entwickelt werden. So wird das Menschsein von Menschen von der Antike bis zur Neuzeit in zwei unterschiedlichen Horizonten bestimmt, insofern die Kategorie des Nichtmenschlichen in die des Göttlichen (Differenz Gott/ Mensch) und des Tierischen (Differenz Mensch/Tier) unterschieden werden kann. Und auch die Menschlichkeit von Menschen wird unterschiedlich bestimmt, je nach dem, ob unter der Negation des Menschlichen das Unmenschliche (das, was dem Menschlichen widerspricht) oder das Nicht-menschliche (das, was nicht menschlich und nicht unmenschlich ist) verstanden wird. Denn Unmensch ist der, der aufgehört hat, Mensch zu sein und als Mensch zu leben, sei es, weil er sich selbst zum Unmenschen macht, indem er sich anderen gegenüber unmenschlich verhält, sei es, indem er zum Unmenschen gemacht wird, indem andere sich ihm gegenüber so verhalten, dass er weder menschlich noch nicht menschlich leben kann. Nicht jeder, der nicht menschlich lebt, lebt unmenschlich. Er kann auch eben das tun, was gesagt wird: nicht menschlich leben. Er lebt nicht als Mensch, ohne ein Unmensch zu sein.

5. Dabei ist eine interessante Asymmetrie zu beachten. Im ersten Fall wird die Wendung «menschliches Leben` als «Leben eines Menschen〉 verstanden und das damit Gemeinte dadurch präzisiert, dass das Menschsein anhand seines Kontrastbezugs zu Göttlichem und/ oder Tierischem bestimmt wird. Im zweiten Fall wird die Wendung «menschliches Leben〉 dagegen als «menschliches Leben eines Menschen verstanden und das damit Gemeinte dadurch präzisiert, dass die Menschlichkeit eines Menschen anhand des Kontrastbezugs zur Nichtmenschlichkeit und Unmenschlichkeit von Menschen be- 
Ingolf U. Dalferth

stimmte wird. Nichtmenschlich lebt ein Mensch, der sich anderen oder sich selbst gegenüber weder menschlich noch unmenschlich verhält, die Menschheit der anderen und seine eigene Menschheit also weder achtet noch sie aktiv beschädigt und bekämpft. Menschlich dagegen lebt ein Mensch, wenn er sich anderen oder sich selbst gegenüber weder unmenschlich (faktisch oder absichtlich menschenverachtend) noch nicht menschlich (die Menschheit bei anderen und bei sich nicht achtend) verhält. Während die Leitdifferenz zur Bestimmung des Menschseins daher ein strenger Dual ist (Mensch/Nichtmensch bzw. menschlich/nichtmenschlich), ist die Leitdifferenz zur Bestimmung der Menschlichkeit ein Trial (menschlich/nichtmenschlich/unmenschlich) und damit ähnlich strukturiert wie die des Vertrauens (Vertrauen/Nichtvertrauen/ Misstrauen bzw. trust/distrust/mistrust).

6. Unter Bedingungen der Neuzeit wird das Menschsein in der Regel nicht im Rekurs auf die metaphysische Unterscheidung von Mensch und Gott am Leitfaden der Unterscheidung endlich/unendlich o.ä. bestimmt, sondern anhand der natürlichen (physischen) bzw. biologischen Differenz zwischen Mensch und Tier, wie sie klassisch in der philosophischen Definition des Menschen als animal rationale zum Ausdruck kommt: Der Mensch teilt mit anderen Lebewesen die Animalität (er ist wie sie ein leiblich-endliches Lebewesen), aber er unterscheidet sich von ihnen durch seine Rationalität (er ist anders als sie ein Lebewesen, das überVernunft verfügt).

7. Beides stellt sich bei genauerer Betrachtung als problematischer heraus, als oft gesehen wird. Auf der einen Seite ist die Animalität keine biologische Gemeinsamkeit, die Menschen mit anderen Tieren als gemeinsame Natur teilen, sondern der Mensch lebt seine biologische Natur auf seine Weise und die Tiere ihre auf ihre Weise. Schon als Tier hat der Mensch Sprache, wie Herder betonte. Deshalb unterscheiden sich Menschen schon in ihrem Tiersein von anderen Tieren und nicht erst in ihrer Rationalität, und sie sind schon in ihrem Tiersein durch ihre Rationalität geprägt und nicht erst im Kontrast zu dieser. Auf der anderen Seite ist auch die Rationalität bzw. Vernunft kein klar bestimmbares Unterscheidungsmerkmal zwischen Mensch und Tier. Auch andere Tiere haben ihre Form von Bewusstsein und ihre Art von Rationalität, und manche Tiere sind in mancher Hinsicht Menschen darin ähnlicher als diese es untereinander sind. Weder benennt die Animalität daher die biologische Gemeinsamkeit von Mensch und Tier (das, was Mensch und Tier biologisch teilen), noch die Rationalität die anthropologische 
Verschiedenheit (das, was den Menschen als Menschen vom Tier als Tier unterscheidet). Beides kann beides manifestieren, und deshalb kann die Differenz zwischen Mensch und Tier nie absolut, sondern im Blick auf die Phänomene der (wie auch immer bestimmten) Animalität und der (wie auch immer gefassten) Rationalität stets nur relativ bestimmt werden. Wir sind den Tieren biologisch näher und anthropologisch ferner, als wir es uns oft zugestehen.

\section{Menschlichkeit}

8. Geht es nicht um die Bestimmung des Menschseins, sondern der Menschlichkeit des Menschen, genügt es nicht, auf die biologische Differenz zwischen Mensch und Tier zu rekurrieren - wie immer diese bestimmt wird. Zur Debatte steht dann vielmehr die anthropologische Differenz zwischen einem menschlichen, nichtmenschlichen und unmenschlichen Leben von Menschen. Menschlich ist ein Leben nicht schon dadurch, dass es das Leben eines Menschen und nicht eines anderen Tieres ist (biologische Differenz Mensch/ Tier), sondern erst dadurch, dass ein Mensch sein Menschsein auf menschliche Weise lebt (anthropologische Differenz menschliches/ nicht menschliches/unmenschliches Leben). Was aber macht das Leben eines Menschen zu einem menschlichen Leben?

9. Eine Antwort darauf - nicht die einzige und keine vollständige oder hinreichende - ist derVerweis aufVertrauen. Kein Mensch lebt menschlich, wenn es kein Vertrauen in seinem Leben gibt, wenn ihm also von anderen nicht vertraut wird (zugemutete Vertrauenswürdigkeit oder erlebtes Vertrauen) und er anderen nicht vertraut bzw. vertrauen kann (Zumutung von Vertrauenswürdigkeit oder gelebtes Vertrauen). Erlebtes und gelebtes Vertrauen sind Kennzeichen eines menschlich gelebten Lebens. Das eine bindet ein in eine menschliche Gemeinschaft (erlebtes Vertrauen), insofern einem von anderen Vertrauen geschenkt und Vertrauenswürdigkeit zugemutet wird. Das andere eröffnet die Chance zu einem menschlichen Zusammenleben (gelebtes Vertrauen), insofern man anderen Vertrauen entgegenbringt und sie für vertrauenswürdig hält - jedenfalls bis zum Erweis des Gegenteils. Das Vertrauen mag enttäuscht werden und ein menschliches Zusammenleben in diesem Fall nicht zustande kommen. Aber wem niemand vertraut, der verkümmert in seinem Menschsein. Und wer anderen nicht vertraut, der bringt damit nicht nur seine Einschätzung ihrer Vertrauenswürdigkeit zum Ausdruck, sondern sein Urteil über ihre Menschlichkeit. Er bestreit 
Ingolf U. Dalferth

nicht, dass sie Menschen sind, aber er behandelt sie als Menschen, mit denen man nicht menschlich zusammenleben kann, weil ihnen - aus welchen Gründen auch immer - nicht zu vertrauen ist. Dieses Urteil mag falsch sein und aus einer Täuschung resultieren, und es kann sich auch wieder ändern oder korrigieren lassen. Aber so oder so bringt es die nicht von ungefähr verbreitete Überzeugung zum Ausdruck, dass ein Mensch menschlich lebt, wenn es erlebtes und gelebtes Vertrauen in seinem Leben gibt, nicht menschlich oder unmenschlich dagegen, wenn es das nicht gibt. Kein Mensch muss auf - vernünftig, also nicht blind, sondern situationsgerecht - vertrauende Weise leben, aber jeder kann es, und nur wenn er es tut, lebt er menschlich. Umgekehrt muss man keinem Menschen vertrauen, aber nur wenn man es tut, behandelt man ihn menschlich.

10. Damit kommt ein wichtiger Sachverhalt in den Blick. Die biologische Differenz zwischen Mensch und Tier steht nicht in unserer Verfügungsmacht: Wir können nicht entscheiden, Mensch oder (ein nichtmenschliches) Tier zu sein. Es gibt kein $\mathrm{x}$, das entweder Mensch oder Tier oder beides oder keines von beiden sein kann, sondern es gibt nur entweder Menschen oder Tiere und weder die einen noch die anderen entscheiden darüber, dass und was sie sind. Wohl aber können wir entscheiden, menschlich oder unmenschlich zu leben, jedenfalls in gewissen Grenzen. Nur wer Mensch ist, kann menschlich leben (insofern setzt die anthropologische Differenz die biologische voraus). Aber wer biologisch Mensch ist, lebt nicht allein deshalb auch schon menschlich - sei es, weil ihm ein solches Leben von anderen unmöglich gemacht wird, oder sei es, weil er es sich selbst versagt und (aus welchen Gründen auch immer) nicht menschlich oder gar unmenschlich mit sich und mit anderen umgeht. Nur Menschen, die leben, können menschlich, nichtmenschlich oder unmenschlich leben. Aber solange sie leben, können sie auch nicht vermeiden, das eine oder das andere davon zu tun.

\section{Verschieden verstandene Menschlichkeit}

11. Man muss nicht von Gott reden, um menschliches Leben im Licht der beiden Differenzmuster menschlich/nichtmenschlich (biologische Differenz) und menschlich/nichtmenschlich/unmenschlich (anthropologische Differenz) zu verstehen. Diese Differenzenfallen auch für die nicht zusammen, die jeden Rekurs auf Gott vermeiden. Was Menschen von (anderen) Tieren unterscheidet, ist eine wissenschaftlich (biologisch) zu klärende Frage. Was menschliches von 
nichtmenschlichem und unmenschlichem Leben unterscheidet, ist dagegen eine geschichtlich in verschiedenen Gesellschaften, Kulturen, Religionen, Moral- und Rechtssystemen nicht einheitlich, sondern unterschiedlich beantwortete Frage. Auch wenn wir heute darum ringen, kulturübergreifende Minimalstandards dessen festzuschreiben, was ein menschenwürdiges Leben von Menschen auszeichnet bzw. auszeichnen sollte (Menschenrechte), ist nicht zu übersehen, dass im Verständnis von «Menschlichkeit» und im Blick auf die Bestimmung dessen, was man für ein menschliches, nicht mehr menschliches oder gar unmenschlichesLebenhält, nicht nur zwischen Kulturen, sondern auch innerhalb derselben Kultur oft grosse Differenzen bestehen.

12. Ebenso wenig ist allerdings $\mathrm{zu}$ übersehen, dass es in jeder Kultur und Gesellschaft lebensorientierende Konzeptionen der Menschlichkeit gibt, die ihre Funktion nur erfüllen, wenn sie auch realisiert und gelebt werden (können). Jede Kultur kennt Exemplare der Menschlichkeit (Vorbilder, Helden, Heilige), denen nachzueifern den Menschen nahegelegt wird und an denen sich die Bemühungen, ein menschliches Leben $\mathrm{zu}$ führen, orientieren. Menschlichkeit kann und muss man lernen. Das erfordert ein Verständnis von Menschlichkeit, das einen leiten kann, und Beispiele seiner Umsetzung, an denen man sich auszurichten vermag. Beides bereitzustellen und an immer wieder neue Generationen zu vermitteln, ist eine gesellschaftlich-kulturelle Aufgabe. Kein Mensch lebt per se schon menschlich. Darum muss man sich bemühen, gemeinsam mit anderen und für sich, und jeder kann dabei scheitern.

13. In einem gehaltvollen Sinn scheitern kann man allerdings nur, wenn so zu leben möglich und nicht unmöglich ist, wenn das Ideal der Menschlichkeit also nicht unerreichbar, aber auch nicht schon längst realisiert, sondern in der richtigen Entfernung vom faktischen Leben angesiedelt ist, nicht zu nahe, aber auch nicht zu fern, sonst hat es kein Orientierungspotential. Es muss möglich sein, im jeweiligen Sinn menschlich zu leben, auch wenn man darüber streiten mag und auch muss, was unter «Menschlichkeit in einem minimalen oder maximalen Sinn verstanden werden sollte. Keine Konzeption von Menschlichkeit ist abschließend und definitiv. Es gibt immer Gründe, sie weiter zu entwickeln, auch wenn es oft lange dauern kann, bis diese erkannt und umgesetzt werden. Menschen können im Horizont ihrer jeweiligen Kultur nicht nur menschlich leben, sondern sie können immer auch noch menschlicher leben. Das Konzept der Menschlichkeit kann und muss fortbestimmt, vertieft 
Ingolf U. Dalferth

und intensiviert werden, und nur solange das geschieht, ist eine Kultur lebendig.

14. Wie und in welcher Hinsicht es weiterentwickelt, vertieft oder intensiviert werden soll, wird in verschiedenen Horizonten und von verschiedenen Standpunkten aus verschieden gesehen. Nicht überall sind es dieselben Schwierigkeiten und Probleme, an denen man sich stösst und die man zu überwinden sucht. Nicht alle halten dieselben Aspekte oder Qualitäten eines Lebens für ein menschenwürdiges oder menschliches Leben wichtig und erstrebenswert. Und nicht jeder sucht das anvisierte Ziel auf einem Weg zu erreichen, der auch andere überzeugt. Auch wenn (in der Regel) niemand dagegen ist, dass Menschen menschlich zusammen leben, ist das, was damit ausgeschlossen sein soll, und das, was man für erstrebenswert hält, oft weit verschieden.

Das Ideal der Menschlichkeit ist keine unstrittige Grösse, und der Streit über die Bestimmung oder Fortbestimmung dieses Ideals ist immer zugleich ein Streit der verschiedenen Standpunkte, von denen aus die Bestimmung erfolgt, und der unterschiedlichen Horizonte, die dadurch ins Spiel kommen. So gibt es naturalistische Konzeptionen, die meinen, auch die anthropologische Frage nach der Menschlichkeit im Horizont der biologischen Frage nach dem Menschsein beantworten zu müssen (reduktionistischer Naturalismus). Umgekehrt gibt es kulturalistische Konzeptionen die meinen, die biologische Frage nach der Differenz von Mensch und Tier zusammen mit der anthropologischen Unterscheidung zwischen Menschlichkeit und Unmenschlichkeit beantworten zu können (reduktionistischer Kulturalismus). Es gibt aber auch einheitsorientierte Konzeptionen, die von der irreduziblen Verschiedenheit der biologischen und anthropologischen Fragestellung ausgehen, ihre differenten Fragen und Antworten aber in einem einheitlichen Zusammenhang aufeinander zu beziehen suchen, der zeichensystematisch (Kulturalismus der symbolischen Formen) oder emergenzmetaphysisch (metaphysischer Naturalismus) entwickelt werden kann. Und schließlich gibt es differenzorientierte Konzeptionen, die nicht nur die Differenz der biologischen und anthropologischen Fragestellungen würdigen, sondern auch sehen, dass diese nicht nur verschieden beantwortet, sondern von verschiedenen Standpunkten aus und in verschiedenen Horizonten auf irreduzibel verschiedene Weise beantwortet werden können, so dass mit irreduzibel pluralen Konzeptionen des Menschseins und der Menschlichkeit zu rechnen ist: Nicht nur die biologische und anthropologische Frage sind irreduzibel verschieden, sondern auch die Standpunkte, von denen 
aus sie beantwortet können, und die Horizonte, in denen sie beantwortet werden.

\section{Theologische Anthropologie}

15. Zu dieser letzten Gruppe gehört die theologische Anthropologie in den Denktraditionen des Christentums. Sie geht im Blick auf die Differenz von menschlichem und nichtmenschlichem Leben davon aus (oder sollte es doch tun), dass Menschen Lebewesen sind, die im Prozess der Entwicklung des Lebens auf der Erde in biologischer und kultureller Ausdifferenzierung von anderen Lebewesen zu dem geworden sind, was sie heute sind. Und sie geht im Blick auf die anthropologische Differenz von menschlichem, nichtmenschlichem und unmenschlichem Leben davon aus (oder sollte es doch tun), dass Menschen nicht religiös oder christlich leben müssen, um menschlich leben zu können. Man muss kein Christ sein, um menschlich zu leben (oder sich doch darum zu bemühen) und andere und sich selbst nicht unmenschlich zu behandeln (oder das doch zu versuchen). Die Menschlichkeit des Lebens ist keine per se religiöse oder gar christliche Kategorie. Aber was es heißen könnte, menschlich zu leben, wo die Grenzlinien zwischen menschlichem, nichtmenschlichem und unmenschlichem Leben zu ziehen sind, und wie man menschlicher leben kann als man gedacht hatte oder sich hätte vorstellen können, darauf geben Christen Antworten, die sich markant von anderen Antworten unterscheiden.

16. Ihre Antwort verdankt sich der Erfahrung, dass Menschen - anderen Menschen und/oder ihnen selbst - in unvorhersehbarer und oft überraschender Weise Möglichkeiten zugespielt werden, die sich nicht aus dem ableiten lassen, was der Fall war und ist, die man nicht erwartet oder erhofft, ja nicht einmal erträumt hatte, sondern die einem die Augen dafür öffnen, dass man in Bezügen zu dem sich selbst erschließenden Ursprung des Möglichen existiert, den Christen «Gott» nennen.

Dass dies der Fall ist, versteht sich nicht von selbst, und man hätte es nicht bemerkt, wenn sich diese Wirklichkeit des Möglichen nicht selbst auf unerwartete und unvorhersehbare Weise als solche zugänglich gemacht hätte. Der paradigmatische Fall dafür ist der Ursprung des christlichen Glaubens, wie er sich im Bekenntnis zur Auferweckung des gekreuzigten Jesus durch Gott ausspricht. $\mathrm{Zu}$ diesem Bekenntnis gehört wesentlich, dass es der Überzeugung der Bekennenden zufolge nicht ihrer eigenen Einsicht oder 
Ingolf U. Dalferth

Fantasie entstammt, sondern dem zu verdanken ist, dessen wirksame Gegenwart sie bekennen. Gott ist nicht nur der, den sie als Ursprung dieser unerwarteten Möglichkeit und ihrer Verwirklichung bekennen, sondern auch der, in dem sie den Ursprung der Wahrnehmung und des Verständnisses dieser Möglichkeit und Wirklichkeit sehen. Deshalb wird Gott nicht nur als der bekannt, der (als 〈Vater〉) den gekreuzigten Jesus (als 〈Sohn〉) in sein göttliches Leben auferweckt hat, sondern auch als der, dem sich (als 〈Geist) der Glaube an die Wahrheit dieses Bekenntnisses verdankt. Die trinitarische Rede von Gott Vater, Sohn und Geist bringt eben das in konzentrierter Verdichtung zum Ausdruck.

\section{Menschlichkeit als Mitmenschlichkeit}

17. Von diesem Punkt aus entwickelt die christliche Theologie all ihre Themen, auch ihr Konzept der Menschlichkeit als göttlich begründete und aus Dankbarkeit gegenüber Gott praktizierte Mitmenschlichkeit. Göttlich begründet ist diese, weil sich das, was im Vollsinn menschlich genannt zu werden verdient, erst im Horizont der Gegenwart dessen erschließt, der selbst diese Gegenwart den Menschen als verborgene Gegenwart ihres sie liebenden Schöpfers erschließt. Nur im Ausgang von dieser Selbsterschließung und nicht unter Absehung von ihr lässt sich das präzisieren, was in christlichem Sinn Menschlichkeit im Vollsinn besagt: als Mensch in der Gegenwart dessen zu leben, der mit seiner Gegenwart Menschen ermöglicht, mit anderen Menschen so zusammen zu leben, dass sie diese und sich selbst als Nächste Gottes verstehen und behandeln können. Wer Gottes Nächster ist, dem - so könnte man mittels der Vertrauensmetapher sagen - vertraut Gott, ohne dass es dafür einen vorgängigen Grund geben würde. Aber indem einem Menschen so Vertrauen entgegengebracht wird, wird ihm zugemutet, sich dieses Vertrauens würdig zu erweisen und seinerseits Gott zu vertrauen und den anderen, denen Gott vertraut - Gott in der Gott gegenüber angemessenen Weise: uneingeschränkt, und den anderen in der ihnen gegenüber angemessenen Weise: vorbehaltlos - jedenfalls so lange diese sich dieses zugemuteten Vertrauens würdig erweisen und ihrerseits Gott und ihren Mitmenschen in der entsprechenden Weise vertrauen. Menschlichkeit wird so als unbeschränkte Mitmenschlichkeit bestimmt und verstanden, und sie wird als solche aus Dankbarkeit dem gegenüber gelebt und praktiziert, der sich jedem Menschen so zuwendet, dass er dessen Nächster wird: Menschlich 
lebt, wer sich jedem Menschen gegenüber so verhält, dass er ihn als einen würdigt, dem Gott selbst sich aus freien Stücken zum Nächsten gemacht hat.

18. Im Rekurs auf diese vorgängige, unverdiente und nicht zu erwartende göttliche Zuwendung steht und fällt das christliche Verständnis von Menschlichkeit und die dadurch geprägte Praxis der Nächstenliebe. Wird von dieser vorgängigen göttlichen Zuwendung abgesehen, bleibt dieses Verständnis von Menschlichkeit als Gott verdankter Mitmenschlichkeit unzugänglich. Genau das aber ist der Normalfall. Menschen verstehen sich und andere nicht als Nächste Gottes, weil sie leben, als gäbe es Gott nicht, ob sie an so etwas wie Gott glauben oder nicht. Ihr Leben orientiert sich nicht an Gottes Zuwendung, sondern allenfalls an dem, was sie für Gott halten, oder an dem sie sich halten, auch wenn sie es nicht 〈Gott〉 nennen. Die einen mögen es für wahrscheinlicher halten, dass es so etwas wie Gott gibt, die anderen, dass es so etwas wie Gott nicht gibt. Beide aber leben ihr Leben so, dass es nicht wesentlich anders aussähe, wenn das Gegenteil von dem wahr wäre, was sie glauben. Das besagt nicht, dass sie auf ihre Weise nicht menschlich leben könnten. Aber ihre Menschlichkeit ist nicht von der Art, um die es in der christlichen Mitmenschlichkeit geht, sie orientieren ihr Leben nicht an Gottes Zuwendung und sie würdigen sich und andere nicht als Nächste Gottes. Damit schöpfen sie - aus christlicher Sicht gesprochen - die Möglichkeiten nicht aus, die ihnen von Gott zugespielt werden, und sie lassen in der Bestimmung und Praxis ihrer Menschlichkeit unausgelotet, was möglich wäre. Ihr Leben muss deshalb nicht schlecht oder menschlich unzulänglich sein. Aber es könnte besser sein und ihre Menschlichkeit als Mitmenschlichkeit realistischer.

19. Von Gottes vorgängiger Zuwendung ist im christlichen Leben ausdrücklich die Rede. Aber diese Zuwendung ist kein Lebensphänomen, sondern das, was die ganze Reihe der Lebensphänomene als Gottes Schöpfung und den Bereich seines Wirkens qualifiziert. Sie ist der Operator, der die Welt zur Schöpfung macht. Als schöpfungsqualifizierender Operator tritt sie nicht selbst innerhalb der Schöpfungswelt phänomenal in Erscheinung. Deshalb ist es nicht natürlich, auf Gottes Zuwendung zu achten, sondern im Gegenteil natürlich, sie nicht zu beachten. Sie ist kein Phänomen der Welt, auf das man im Zusammenhang seiner Lebenserfahrungen neben oder unter anderen früher oder später stossen würde, müsste oder könnte. Sie ist vielmehr der nichtphänomenale Orientierungspunkt einer Lebensweise, in der man lernt, sich selbst als Geschöpf 
Ingolf U. Dalferth

unter Geschöpfen zu verstehen und die Welt und ihre Phänomene als Schöpfungzu erleben.

20. Auf diesen Orientierungspunkt und die ihm korrelierte Lebensweise wird man nur aufmerksam, wenn man von sich selbst und vom normalen Lebensprozess so distanziert wird (existentielle Dislozierung), dass man die bisherige Reihe nicht einfach fortsetzt, sondern einen anderen und neuen Blick auf alles gewinnt (existentielle Neuorientierung). Diese Distanzierung kann man nicht selbst veranlassen, denn dann wäre sie eine Fortsetzung des Bisherigen. Sie resultiert vielmehr aus einer überraschend widerfahrenden oder sich langsam anbahnenden fundamentalen Unterbrechung des Lebenszusammenhangs, die sich selbst und alle Erfahrungen und Phänomene neu zu sehen und anders zu verstehen nötigt: nicht nur als Welt, sondern als Schöpfung, nicht nur als Phänomene, sondern als Zeichen, die auf den Schöpfer verweisen (bonum) oder diesen Verweis verdunkeln und verstellen (malum), nicht nur als Menschen, sondern als Geschöpfe, die in und durch Gottes Gegenwart leben.

21. Existentielle Dislozierung und Neuorientierung ereignen sich wenn überhaupt - im menschlichen Leben. Sie haben daher immer ein Vorher, in dem das Leben anders gelebt wurde. Auch in diesem vorherigen Leben stehen Menschen vor der Herausforderung, sich zur Menschlichkeit zu entscheiden. Die Entscheidung, menschlich und nicht nichtmenschlich oder unmenschlich zu leben, wird dabei aber - theologisch geurteilt - von Menschen getroffen, die leben, als gäbe es Gott nicht, obwohl es sie doch nicht gäbe, wenn Gott nicht mit ihnen und für sie lebte. Sofern das der Fall ist, ignorieren sie einen grundlegenden Aspekt ihrer Lebenswirklichkeit und schöpfen die Möglichkeiten der Menschlichkeit nicht aus, weil sie Gottes Gegenwart in ihrem Leben ausblenden. Das ändert nichts an der Wirklichkeit dieser Gegenwart, wohl aber bestimmt es die Einstellung der Menschen zu ihr und damit die Adäquatheit der Wahrnehmung ihrer realen Lebenssituation. Weil sie ihr Leben nicht als Ort der Gegenwart Gottes wahrnehmen, verkennen sie sein Potential und unterschätzen ihre Möglichkeiten. Sie leben - theologisch gesprochen - als «Sünder〉 und wissen es nicht.

Das ist keine moralische Abqualifizierung von Menschen, sondern Kurzformel ihrer unaufgeklärten Situation im Horizont der Gegenwart Gottes. So blind beginnt das Leben jedes Menschen, und bei den meisten endet es auch so. Die Entscheidung zwischen einem menschlichen, nichtmenschlichen und unmenschlichen Leben wird dementsprechend ohne Beachtung dessen gefällt, ohne den 
es weder Menschen noch die Möglichkeit eines menschlichen oder nichtmenschlichen bzw. unmenschlichen Lebens gäbe. Was «Menschlichkeit heißen kann, bleibt so unausgelotet: Als Gott verdankte Mitmenschlichkeit kommt sie nicht in den Blick.

22. Nicht primär zur Bestimmung des Menschseins ist der Bezug auf Gott daher wichtig, wie die Tradition angenommen hat, sondern zur Bestimmung der Menschlichkeit des Menschen. Wer nicht mit Gottes guter Gegenwart in seinem Leben und dem der anderen rechnet, hört nicht auf, Mensch zu sein, er ist auch kein Unmensch oder unfähig, menschlich zu leben. Aber er weiß auch nicht, was menschlich zu leben im Vollsinn heißen könnte, weil er sich in der Gestaltung seines Lebens nur im Bezug auf andere und anderes orientiert, aber den Bezug auf Gott ignoriert oder ausblendet, ohne den es weder anderes noch andere noch ihn selbst gäbe. Auch Sünder können menschlich leben. Aber sie leben mit einem Aufklärungsdefizit und damit unter den Möglichkeiten, die Menschen zur Gestaltung ihrer Menschlichkeit eigentlich offenstünden. Und da alle Menschen Sünder sind, leben alle mit dieser Beschränktheit.

\section{Vertrauen als Phänomen der Mitmenschlichkeit}

23. Wird Vertrauen vor diesem differenzierten Hintergrund zu verstehen gesucht, dann lässt es sich einerseits am Leitfaden der biologischen oder der anthropologischen Differenz (biologisches vs. anthropologisches Verständnis) näher bestimmen, andererseits können diese Differenzen entweder von nichttheologischem oder von theologischem Standpunkt aus (nichttheologisches vs. theologisches Verständnis) entfaltet werden.

24. In biologischer Hinsicht wird Vertrauen als ein Phänomen des menschlichen und nichtmenschlichen (bzw. gewisser Formen nichtmenschlichen) Lebens in den Blick gefasst, das sich empirisch beschreiben lässt, insofern bestimmte Verhaltensweisen (trustingbehavior) mit bestimmten Gehirnaktivitäten (der Aktivierung bestimmter neuronaler Netzwerke) korrelieren. Vertrauen verstehen heißt dementsprechend, die neurobiologischen Grundlagen von vertrauendem Verhalten zu verstehen - sei es bei Menschen, bei Katzen oder bei Hunden.

25. In anthropologischer Hinsicht führt dieser Weg zu keinen Einsichten. Wird Vertrauen als anthropologisches Phänomen zu verstehen versucht, dann muss es im Horizont der Differenz von 
Ingolf U. Dalferth

menschlichem, nichtmenschlichem und unmenschlichem Verhalten verstanden werden. Diese Differenz tritt nur im menschlichen Leben auf, und sie kann nicht expliziert werden ohne Rekurs auf eine Idee der Menschlichkeit, d.h. eine Vorstellung oder Konzeption dessen, was menschliches Leben menschlich und unmenschliches Leben unmenschlich macht. Biologische Antworten greifen da zu kurz, weil sie zwar Aspekte des Menschseins, aber nicht der Menschlichkeit, also den menschlichen, nichtmenschlichen oder unmenschlichenVollzug des Menschseins verständlich machen können. Um das zu tun, muss man Vertrauen anders in den Blick fassen, nicht nur biologisch-natürlich als Sichverlassen auf andere oder anderes oder als in Anspruch genommene Verlässlichkeit der Eltern-Kind-Beziehungen im Rahmen des Herden- oder Gruppenverhaltens von Menschen, sondern personal-kulturell als reziprokes und selbstreferentielles Vertrauen in die, die einem vertrauen, und die damit auch darauf vertrauen, dass auch ihnen vertraut und ihr Vertrauen nicht enttäuscht wird.

26. Doch auch das ist noch ein verkürztes Verständnis, weil es Vertrauen auf die zu beschränken scheint, die einem vertrauen, während alle anderen davon ausgeschlossen sind. Wird Vertrauen als Moment konkret gelebter Menschlichkeit verstanden, und ist diese ein Modus des Lebens, der allen Menschen offen steht und zuzumuten ist, dann muss die Reichweite des Vertrauens über das bisher Gesagte hinaus entschränkt werden. Eben das unterstreicht, dass sich das anthropologische Verständnis nicht auf der Basis des biologischen Verständnisses entwickeln lässt. Biologisch ist nicht einsichtig zu machen, dass man nicht nur andere im familiären und freundschaftlichen Nahbereich (Gruppenvertrauen in Familie, Clan, Freundeskreis), sondern alle Menschen lieben, als Menschen würdigen, vertrauensvoll behandeln soll. Diese nicht-natürliche Haltung kann nicht biologisch begründet, sondern nur anthropologisch als prekäre, gefährdete und nie sich von selbst verstehende «zweite Natur des Menschen entfaltet werden. Nicht von ungefähr tritt sie in dieser universalen Entschränkung nicht in allen, sondern nur in einigen wenigen Kulturen auf. Und sie ist auch in diesen anthropologisch kein selbstverständliches, sondern ein äusserst unselbstverständliches Phänomen.

27. Dass sich der Sinn von «Menschlichkeit» nicht im Blick auf einen Menschen allein, sondern immer nur im Zusammenhang menschlicher Gemeinschaft und Kultur bestimmen lässt, ist dabei vorausgesetzt. Es ist ein nicht-natürlicher Modus des Sichverhaltens 
anderen und sich selbst gegenüber, in dem die Möglichkeiten des Menschseins andererund ihr Recht, sie auf ihre Weise zu leben, nicht weniger geachtet werden als die eigenen. Wer menschlich lebt, kann sich nicht anderen gegenüber unmenschlich und sich selber gegenüber menschlich verhalten, oder umgekehrt. Man verwickelt sich in Selbstwidersprüche und Verkürzungen im Bemühen um Menschlichkeit, wenn man der Maxime derWürdigung des Menschseins von Menschen im eigenen Leben nicht uneingeschränkt folgt, sondern Ausnahmen macht, also einige andere (die einem Nahestehenden) menschlich und andere - aus welchen Gründen auch immer - nichtmenschlich oder unmenschlich behandelt.

Gerade das freilich ist gang und gäbe. Es versteht sich deshalb auch keineswegs von selbst, dass man menschlich lebt, sondern das bedarf einer besonderen Bemühung. Wer sich wirklich menschlich verhalten will, der kann sich nicht damit begnügen, seine Freunde freundlich und andere anders zu behandeln. Er ist vielmehr herausgefordert, den Möglichkeiten eines Menschen (den Möglichkeiten anderer Menschen und den eigenen Möglichkeiten) möglichst uneingeschränkt gerecht $\mathrm{zu}$ werden - möglichst uneingeschränkt, weil die Sicht der Möglichkeiten des anderen und seiner selbst stets durch den eigenen Standpunkt und den dadurch bedingten Horizont begrenzt ist. Auch wenn wir alles zu sehen meinen, sehen wir nicht alles, weil wir nur sehen können, was von unserem Standpunkt aus gesehen werden kann. Das ist meist mehr als wir meinen, aber es ist niemals genug oder gar alles.

\section{Vertrauen als christliche Lebenspraxis}

28. An dieser Stelle zeigt sich die Wichtigkeit des Standpunkts, von dem aus man Vertrauen zu leben und/oder zu verstehen sucht, und damit der Differenz zwischen einem nichttheologischen und einem theologischen Standpunkt. In beiden Horizonten können Phänomene der Menschlichkeit und des Vertrauens thematisiert werden, aber sie werden jeweils in anderem Licht und in anderer Hinsicht betrachtet. Die Grunddifferenz ist, dass von nichttheologischem Standpunkt aus der Gottesbezug keine maßgebliche Rolle spielt, während er den theologischen Standpunkt definiert. Deshalb ist der Wechsel vom einen zum anderen nicht nur ein Standpunktwechsel, der andere Phänomene derselben Art in den Blick kommen lässt, sondern ein Wechsel der Weltsicht, der alle 
Ingolf U. Dalferth

aktuellen und möglichen Phänomene in einem anderem Horizont thematisiert.

29. In theologischem Horizont wird Vertrauen dementsprechend nicht nur als Phänomen der Menschlichkeit, sondern der als unbeschränkte Mitmenschlichkeit konkretisierten Menschlichkeit verstanden. Für Christen gilt - und das ist eine 〈grammatische〉 Feststellung, der im konkreten Vollzug des Lebens nur all zu oft widersprochen wird -, dass sie nicht nur denen vertrauen, die ihnen vertrauen, sondern allen Menschen mit Vertrauen begegnen, weil sie darauf setzen, dass ihnen der vertraut, der auch allen anderen vertraut, obwohl diese ebenso wenig Anlass dafür bieten wie sie selbst. Die Vorgabe der bedingungslosen vertrauenden Zuwendung Gottes zu uns wird als Anlass und Grund verstanden, auch anderen ohne Vorbedingungen mit Vertrauen zu begegnen, da Gott auch ihnen als seinen Nächsten Vertrauen entgegenbringt. Menschlichkeit wird als vertrauende Mitmenschlichkeit praktiziert, weil sie aus einer Vertrauensvorgabe lebt, die in Anspruch genommen, aber niemals eingeholt werden kann.

30. Weil sich dieses mitmenschliche Vertrauen nicht aus der Vertrauenswürdigkeit der anderen begründet (wir vertrauen anderen, weil und insofern sie vertrauenswürdig sind), sondern aus der vertrauenden Zuwendung Gottes zu denen, die wie wir selbst nicht vertrauenswürdig sind, ist die stets zu erwartende Enttäuschung des in andere und uns selbst gesetzten Vertrauens kein hinreichender Grund, vertrauende Mitmenschlichkeit nicht mehr zu praktizieren. So gelebte Mitmenschlichkeit muss nicht daran scheitern, dass das Vertrauen in andere faktisch enttäuscht wird. Natürlich kann und wird das immer wieder geschehen. Aber die christliche Haltung und Praxis unbeschränkter Mitmenschlichkeit ist durch dieses faktische Scheitern nicht widerlegt oder außer Kraft gesetzt. Damit rechnet sie vielmehr jederzeit, und dem widersetzt sie sich von Anfang an kontrafaktisch. Sie setzt gerade angesichts enttäuschten Vertrauens auf Vertrauen. Sie kann das, weil sie in anderem gründet und deshalb nicht abhängig davon ist, dass andere oder wir selbst das angetragene Vertrauen nicht enttäuschen. Christlich wird Vertrauen stets angesichts faktischer Vertrauensenttäuschung praktiziert - von Versagern mit Versagern. So gelebtes Vertrauen ist Widerspruch und Einspruch gegen das, was menschliches Leben normalerweise auszeichnet, und es ist eben so der praktische Hinweis darauf, dass menschliches Leben anders sein könnte als es ist.

31. Vertrauen ist menschlich. Aber wäre es nur das, würde es da enden, wo es enttäuscht wird. Um auf enttäuschtes Vertrauen nicht 
nur mit Vertrauensentzug und Vertrauensenthaltung zu reagieren, sondern ohne die Enttäuschung zu beschönigen trotz allem weiter und neu auf Vertrauen zu setzen, erfordert eine Begründung der Lebensorientierung unbeschränkter vertrauender Mitmenschlichkeit, die sie sich nicht an deren faktischem Erfolg oder Misserfolg bemisst oder von diesem abhängig macht. Wer nur denen meint vertrauen zu können, die ihm vertrauen, führt ein einsames Leben, weil er nie sicher sein kann, dass andere ihm tatsächlich vertrauen. Ein universaler Modus des Lebens kann Vertrauen nur werden, wenn es nicht auf faktische Bestätigungen angewiesen ist (so sehr es auf diese hoffen wird), sondern kontrafaktisch gelebt werden kann, weil es sein Recht und seine Kraft nicht aus dem Verhalten der anderen oder dem eigenen Verhalten herleitet, sondern in der Nichtselbstverständlichkeit einer vorausgehenden Zuwendung gründet, mit der angesichts der eigenen Lebenswirklichkeit nicht $\mathrm{zu}$ rechnen ist. Wer so vertraut, der vertraut als einer, der weiss, dass ihm und anderen nicht zu vertrauen ist. Er vertraut wider besseren Wissens, weil ihm vertraut wird und sich dadurch überhaupt erst die Möglichkeit eröffnet, sich vertrauenswürdig zu erweisen. Niemand muss das tun. Aber jeder kann es, auch dann, wenn andere es nicht tun. So hervorgelocktes Vertrauen ist frei, und nur freies Vertrauen ist frustrationsresistent und steht und fällt nicht damit, dass es auf Widerhall stösst. In einer Welt des Misstrauens, Nichtvertrauens und enttäuschten Vertrauens so frei zu vertrauen, ist nicht von jedem Standpunkt aus möglich und einsichtig. Von christlichem Standpunkt aus sollte nichts anderes möglich und einsichtig sein. 